\title{
Cigarette Smoking Exposure and Heart Failure Risk in Older Adults: The Health, Aging, and Body Composition Study
}

\author{
Deepa M. Gopal, MD, MS(1), Andreas P. Kalogeropoulos, MD, PhD(2), Vasiliki V. \\ Georgiopoulou, MD ${ }^{(2)}$, Andrew L. Smith, MD(2), Douglas C. Bauer, MD(3), Anne B. Newman, \\ MD, MPH ${ }^{(4)}$, Lauren Kim, MD(5), Kirsten Bibbins-Domingo, MD(3), Hillary Tindle, MD ${ }^{(4)}$, \\ Tamara B. Harris, MD, MS(5), Wilson W. H. Tang, $\mathbf{M D}^{(6)}$, Stephen B. Kritchevsky, $\mathbf{P h D}^{(7)}$, and \\ Javed Butler, MD, MPH ${ }^{(2)}$ for the Health ABC Study \\ (1)Boston University, Boston, MA \\ (2)Emory University, Atlanta, GA \\ (3)University of California San Francisco, San Francisco, CA \\ (4)University of Pittsburgh, Pittsburgh, PA \\ (5)National Institute of Aging, National Institutes of Health, Bethesda, MD \\ ${ }^{(6)}$ Cleveland Clinic Foundation, Cleveland, $\mathrm{OH}$ \\ (7)Wake Forest University, Winston-Salem, NC
}

\section{Abstract}

Background-Although there is evidence linking smoking and heart failure (HF), the association between lifetime smoking exposure and HF in older adults and the strength of this association among current and past smokers is not well known.

Methods-We examined the association between smoking status, pack-years of exposure and incident HF risk in 2, 125 participants of the Health, Aging, and Body Composition Study (age 73. $6 \pm 2.9$ years; $69.7 \%$ females; $54.2 \%$ whites)using proportional hazard models.

Results-At inception, $54.8 \%$ of participants were non-smokers, $34.8 \%$ were past smokers, and 10. $4 \%$ were current smokers. During follow-up (median, 9.4 years), HF incidence was 11.4 per 1000 person-years in non-smokers, 15. 2 in past smokers (hazard ratio [HR] vs. non-smokers 1. 33; 95\% confidence interval [CI] 1. 01, 1. 76; $\mathrm{p}=0.045$ ), and 21. 9 in current smokers (HR 1. 93; 95\% CI 1. 30, 2. 84; $\mathrm{p}=0.001$ ). After adjusting for HF risk factors, incident coronary events, and competing risk for death, a dose-effect association between pack-years of exposure and HF risk was observed (HR 1. 09; 95\% CI 1. 05, 1. 14; $\mathrm{p}<0.001$ per 10 pack-years). HF risk was not modulated by pack-years of exposure in current smokers. In past smokers, HR for HF was 1.05 (95\% CI, 0. 64, 1. 72) for 1-11 pack-years; 1.23 (95\% CI, 0. 82, 1. 83) for 12-35 pack-years; and $1.64(95 \% \mathrm{CI}, 1.11,2.42)$ for $>35$ pack-years of exposure in fully adjusted models ( $\mathrm{p}<0.001$ for trend) compared to non-smokers.

(C) 2012 Mosby, Inc. All rights reserved.

Address correspondence to: Javed Butler, MD MPH, Emory Cardiovascular Clinical Research Institute, 1462 Clifton Road NE, Suite 504, Atlanta, GA 30322 Telephone No: 404-778-5273 Fax No: 404-778-5285 javed.butler@emory.edu.

Industry Disclosures: None

Publisher's Disclaimer: This is a PDF file of an unedited manuscript that has been accepted for publication. As a service to our customers we are providing this early version of the manuscript. The manuscript will undergo copyediting, typesetting, and review of the resulting proof before it is published in its final citable form. Please note that during the production process errors may be discovered which could affect the content, and all legal disclaimers that apply to the journal pertain. 
Conclusions-In older adults, both current and past cigarette smoking increase HF risk. In current smokers, this risk is high irrespective of pack-years of exposure, whereas in past smokers there was a dose-effect association.

\section{INTRODUCTION}

Cigarette smoking is one of the most preventable causes of morbidity and mortality globally. In North America, 650, 000 deaths are attributable to smoking annually and account for nearly $25 \%$ of total adult mortality, with cardiovascular diseases contributing to $42.1 \%$ of these deaths. ${ }^{1}$ Cigarette smoking leads to impaired endothelial function via decreased nitric oxide production, pro-thrombotic state, increased oxidative stress, and activated inflammatory pathways. ${ }^{2-5}$ Not surprisingly, therefore, smoking is a major contributor to all forms of cardiovascular disease. ${ }^{6}$ In addition to vascular effects, smoking, via increased oxidative stress and inflammation, ${ }^{6,7}$ directly effects on the myocardium leading to systolic and diastolic dysfunction. ${ }^{8,9}$ It also promotes other heart failure (HF) risk factors including blood pressure, increased heart rate, diabetes, and atherosclerosis. ${ }^{10-12}$ We have recently shown that smoking is independently associated with 5-year risk for HF in older adults. ${ }^{13}$ An association between cumulative smoking exposure (in terms of pack-years) and cardiovascular endpoints has been described. ${ }^{14-17}$ However, it remains unclear whether this association is true for HF also. Although the evidence linking smoking and HF is known, the nature of this association with respect to cumulative lifetime smoking exposure in older adults, and the strength of association among current versus past smokers, is not well known. Also, whether this association is independent of incident coronary events is not known either. Finally, in older adults, risk for cardiovascular events may be confounded by the high mortality rate; most studies have not taken into account the competing risk of death. In this study, we assessed the association between smoking status and HF risk among the elderly participants of the Health, Aging, and Body Composition (Health ABC) Study; exploring in particular the cumulative life-time exposure and the related differences in risk between past versus the current smokers, adjusting for incident coronary events and competing death risk.

\section{METHODS}

\section{Study population and baseline data collection}

The Health Aging and Body Composition (Health ABC) Study is a cohort of 3, 075 wellfunctioning, community-dwelling men and women aged 70-79 years at inception. Potential participants were recruited from a random sample of white and all black Medicare beneficiaries residing in designated zip code areas in Pittsburgh, PA, and Memphis, TN, with a mailed invitation followed by a telephone-screening interview to determine eligibility. Recruitment period was from March 1997 to July 1998. Exclusion criteria included difficulty performing basic activities of daily living or walking $1 / 4$ of a mile or climbing 10 steps without resting, use of a cane, walker, crutches, or other equipment for movement, treatment for cancer in the previous three years, intention to move out of the area in the next three years, or participation in a trial involving lifestyle intervention. Eligible participants were scheduled for a home interview during which eligibility was confirmed, written informed consent was obtained, and a comprehensive interview was conducted followed by a clinic examination that included assessment of mobility. The Institutional Review Boards at both sites approved the protocol. Physiologic measurements, blood samples, performance measurements, and questionnaires were obtained during a baseline clinic visit in the two field centers. Subsequently, follow-up data collection and surveillance was conducted by in-person examination alternating with a telephone interview every 6 months. 
Participants with prevalent HF, possible HF, or missing data on HF were excluded ( $\mathrm{n}=140$ ) from this analysis. Of the 2935 participants without prevalent HF, 2890 had smoking data at baseline. Overall 765 participants indicated cigar use; there was a considerable overlap among current, past, and non-cigarette smokers with respect to cigar habit. Due to this overlap and no credible method to equate a specific unit of cigar consumption with equivalent cigarette smoking pack-year exposure, all cigar users $(n=765)$ were excluded from the primary analysis, which was restricted to 2, 125 participants. Cigar smoking was controlled for in an a priori planned secondary analysis.

\section{Study definitions}

Cardiovascular disease status at baseline, including prevalent heart failure, was based on self-reported history, use of selected drugs, and International Classification of Diseases, Ninth Revision, Clinical Modification codes as reported by Medicare and Medicaid Services from 1995 through 1998. Smoking status was classified as current, past ( $\geq 100$ lifetime cigarettes), or non-smoker. Smoking exposure, expressed as pack-years, was calculated as the average number of packs of cigarettes smoked per day (defining pack as 20 cigarettes) times the number of years of smoking. All smoking data were obtained through participant self-report at the baseline visit; secondhand smoking exposure data were not available in this study. Race was self-defined by the participant. Diabetes mellitus was defined as a selfreported history of diabetes mellitus or use of anti-diabetic medication. Left ventricular hypertrophy was ascertained by the electrocardiogram using the following voltage criteria: $\mathrm{R}$ amplitude $>26 \mathrm{~mm}$ in either $\mathrm{V}_{5}$ or $\mathrm{V}_{6}$, or $\mathrm{R}$ amplitude $>20 \mathrm{~mm}$ in any of leads I, II, III, a $\mathrm{VF}$, or $\mathrm{R}$ amplitude $>12 \mathrm{~mm}$ in lead aVL or $\mathrm{R}$ amplitude in $\mathrm{V}_{5}$ or $\mathrm{V}_{6}$ plus $\mathrm{S}$ amplitude in $\mathrm{V}_{1}>35 \mathrm{~mm}$. Coronary heart disease was defined as: (1) history of surgical or percutaneous revascularization; or (2) electrocardiographic evidence of myocardial infarction; or (3) selfreported history of myocardial infarction or angina accompanied by use of anti-anginal medications. Hypertension was defined as self-reported history of physician diagnosis accompanied by use of antihypertensive medications. Incident coronary events were defined as hospitalization for myocardial infarction or angina pectoris, or elective surgical or coronary revascularization.

\section{Follow-up and outcomes}

Participants were asked to report any hospitalizations and were also asked direct questions regarding incident cardiovascular events during the planned telephone interviews and inperson examinations. Medical records for overnight hospitalizations were reviewed at each site by local adjudicators. All first admissions with an overnight stay that was confirmed as related to HF, based on symptoms, signs, chest radiograph results, and echocardiographic findings, using criteria similar to those used in the Cardiovascular Health Study, were designated as incident HF event. ${ }^{18}$ The criteria required HF diagnosis by a physician and treatment for HF. ${ }^{19}$ Briefly, an HF event was confirmed if, in addition to a physician diagnosis, there was documentation in the medical record of (1) symptoms (e. g. , shortness of breath, fatigue, orthopnea) and physical signs (e. g. edema, rales); (2) supporting clinical findings (e. g. pulmonary edema on chest x-ray examination) and (3) HF treatment, including diuretics, digitalis, ACE inhibitors or beta-blockers. Information from echocardiography was not required, but was taken into account whenever available. Incident coronary events were identified and adjudicated using the standard Health ABC Study surveillance and adjudication process described above. All deaths were reviewed by the Health ABC Diagnosis and Disease Ascertainment Committee. Left ventricular ejection fraction post-HF development was abstracted from echocardiography or left ventriculography reports during the index hospitalization; these procedures were performed at the discretion of the treating physician and were therefore available in a subset of patients. 
We used a cut-off point of $45 \%$ to define HF with reduced ( $\$ 45 \%$ ) vs. preserved ( $>45 \%$ ) ejection fraction. ${ }^{20}$

\section{Statistical analysis}

Descriptive statistics are presented as mean (standard deviation) for continuous and number (percentage) for categorical variables. Baseline characteristics between participants were compared with nonparametric methods; utilizing the Kruskal-Wallis test for continuous and $\chi^{2}$ test for categorical variables. Correlation between HF risk factors and smoking variables was examined by Spearman rank correlation. The association between smoking status, cigarette exposure (pack-years)and HF risk was examined with Cox models. Smoking exposure (pack-years) was examined as a continuous variable and as acategorized variable using tertiles of pack-years. We examined for non-linear association between pack-years and HF risk using fractional polynomials and restricted cubic splines. The proportional hazards assumption was evaluated by examining log-log survival plots and interaction terms with time in Cox models.

In multivariable models, we adjusted for other previously identified clinical predictors of $\mathrm{HF}$ in the Health ABC Study (age, coronary artery disease, left ventricular hypertrophy, systolic blood pressure, heart rate, albumin, fasting glucose, and creatinine $)^{13}$ and incident coronary events (defined as incident myocardial infarction, angina, or coronary revascularization, either percutaneous or surgical). We repeated the analyses using the Fine and Grey extension of the Cox model ${ }^{21}$ as a sensitivity analysis to account for death as a competing risk in this older adults cohort. Standard Cox models tend to overestimate the risk of the event of interest (in this study, HF) because participants who died prior to development of $\mathrm{HF}$ are censored. Competing risk analysis estimates disease incidence in the presence of the competing risk by assessing probability of disease conditional on disease-free survival and competing risk-free survival. ${ }^{22}$

In secondary analyses, the association between pack-years of exposure and HF risk was assessed among, (a) past versus current smokers; (b) in race-based sub-groups, and (c) for those who developed HF with preserved versus reduced ejection fraction. Analyses were also repeated in a cohort including participants who reported cigar smoking by controlling for cigar habit. A two-sided $\mathrm{p}<0.05$ was considered significant. Analyses were performed with SAS 9. 2 (SAS Institute Inc., Cary, NC).

\section{RESULTS}

\section{Baseline participant characteristics}

Of the 2, 125 eligible persons, 1, 165 (54. 8\%) were non-smokers, 221 (10.4\%) were current smokers and 739 (34.8\%) were past smokers. Among participants with smoking history, tertiles of pack-year smoking exposure was categorized as lowest (1-11), intermediate (12-35), and highest (>35) tertile. Table 1 shows the baseline participant characteristics by smoking exposure. Mean age of participants was $73.6 \pm 2.9$ years; $69.7 \%$ were females and $54.2 \%$ were white.

\section{Smoking status and heart failure risk}

Over a median follow-up of 9.4 years (interquartile range, 7.0 to 9.4 years), 231 (10. 9\%) participants developed HF (13. 6 events per 1000 person-years). Incidence of HF was 11.4 events per 1000 person-years in non-smokers, 15.2 events per 1000 person-years in former smokers (hazard ratio [HR] vs. non-smokers 1. 33; 95\% confidence interval [CI] 1. 01, 1. 76; $\mathrm{p}=0.045$ ), and 21.9 events per 1000 person-years in current smokers (HR vs. nonsmokers $1.93 ; 95 \%$ CI 1. 30, 2. 84; $p=0.001$ ), Figure 1 . The increased risk associated with 
smoking persisted in models adjusting for previously identified HF risk factors in the Health ABC Study (age, coronary artery disease, left ventricular hypertrophy, systolic blood pressure, heart rate, albumin, fasting glucose, and creatinine), incident coronary events, and competing risk for death. The increased risk associated with smoking persisted in models adjusting for previously identified HF risk factors in the Health ABC Study (age, coronary artery disease, left ventricular hypertrophy, systolic blood pressure, heart rate, albumin, fasting glucose, and creatinine), incident coronary events, and competing risk for death. The adjusted HR was 1.73 (95\% CI 1. 15, 2. 59, $\mathrm{p}=0.008)$ for current smokers and $1.31(95 \%$ CI $0.98,1.75 ; \mathrm{p}=0.06$ ) for past smokers, versus non-smokers.

\section{Cigarette smoking exposure and heart failure risk}

We did not detect any high-order (nonlinear association between pack-years and HF risk. When pack-years were evaluated as a continuous variable in unadjusted models, a significant association with HF risk was observed (HR 1. 09;95\% CI 1. 05, 1. 14; p<0. 001 per 10 pack-years). This dose-effect association was only evident among past smokers (HR 1. 11;95\% CI 1. 05, 1. 18; $\mathrm{p}<0.001$ per 10 pack-years). There was no dose-effect association in current smokers (HR 0. 94;95\% CI 0. 82, 1. 08). When pack-years exposure was assessed as tertiles in past smokers, there was an increase in risk with increasing exposure(1-11 packyears: 9.1 events; $12-35$ pack-years: 16.8 events; and greater than 35 pack-years: 20.6 events per 1000 person-years), Figure 2. In multivariate models adjusting for previously identified HF risk factors in Health $\mathrm{ABC}$, incident coronary events, and competing risk for death, the dose-effect in past smokers across tertiles was 1.05 (95\% CI 0. 64, 1. 72; lowest), 1. 23 (95\% CI 0. 82, 1. 83; intermediate), and 1.64 (95\% CI 1. 11, 2. 42; highest tertile), Table 2. Secondary analysis including participants who reported cigar use revealed persistent dose-effect association of pack-years with HF (HR 1. 06, 95\% CI 1. 02, 1. 09; p = 0.003 per 10 pack-years, controlling for HF risk factors, incident coronary events, competing risk for death, and cigar use).

\section{Subgroup analyses}

In the combined subgroups of current and past smokers, the relationship between cigarette exposure and HF risk in unadjusted models was comparable in whites(HR 1. 12; 95\% CI 1. 07, 1.18 per 10 pack-years exposure vs. nonsmokers; $p$ < 0. 001)and blacks(HR 1. 06; $95 \%$ CI $0.99,1.14$ per 10 pack-years vs. nonsmokers; $\mathrm{p}=0.11$ ); $\mathrm{p}=0.26$ for interaction. Similarly, the association of cigarette smoking and incident HF risk in females and males were comparable (HR 1. 06; 95\% CI 1. 00, 1. 13; $\mathrm{p}=0.058$ and HR 1. 12; 95\% CI 1. 06, 1. $19 ; \mathrm{p}<0.001$ per 10 pack-years for females and males respectively; interaction $\mathrm{p}=0.19$ ). Finally, quit time had no effect on the association between pack-years and HF risk among past smokers.

Information on left ventricular ejection fraction post-HF was available in 179 of 231 (77. $5 \%$ ) cases. There were 80 cases $(44.7 \%)$ with preserved ejection fraction. HF risk in fully adjusted models stratified by those with preserved or depressed ejection fraction was not different (HR 1. 05; 95\% CI 0. 969, 1. 14 vs. HR 1. 11; 95\% CI 1. 04, 1.18 per 10 packyears respectively, $\mathrm{p}$ value for $z$ test $=0.34$ ).

\section{DISCUSSION}

In this study, current smokers exhibited a significantly increased risk of incident HF compared to non-smokers after controlling for other clinical HF risk factors and incident coronary events. Interestingly, there was no dose-effect association between cigarette exposure and risk for HF in current smokers on this risk. In contrast, pack-years of exposure in past cigarette smokers modulated HF risk. These associations persisted in models 
accounting for the competing risk of death in this older adult cohort. These results highlight not only the importance of current and past smoking in terms of risk for HF in older adults, but also underscore the fact that there is no 'safe' level of active smoking for HF risk.

The propagation of atherosclerosis and myocardial infarction by cigarette smoking is well delineated. The nature of the association between cigarette smoking and HF risk has not been defined clearly in older adults. We demonstrated an increase in HF risk with cigarette smoking, which was higher for current vs. past smokers. Interestingly, the association of smoking with HF risk persisted despite adjustment for other presumptive causal pathways, such as other clinical HF risk factors and incident coronary events. The mechanism by which smoking promotes atherosclerosis appears to be mediated by inflammation. 5, 23 Heart failure risk with smoking persists after controlling for inflammatory markers, suggesting that alternate mechanisms may also be important. ${ }^{24,25}$ Cigarette smoking directly increases free radical production, ${ }^{26}$ cardiac mitochondrial damage, ${ }^{27}$ and nicotineinduced free fatty acid release ${ }^{28}$; all well-described pathways of HF development. To what extent these alternate mechanisms provide the link between cigarette smoking and HF risk remains to be elucidated.

We observed a dose-effect relationship between pack-years of exposure and HF risk in past smokers. Previous studies have demonstrated a continuous relationship between pack-years of exposure and carotid artery intima-media thickness, implying a cumulative adverse effect on vascular structure. ${ }^{29} \mathrm{~A}$ dose-response relationship between cigarette smoking and left ventricular systolic function in asymptomatic individuals has also been shown. ${ }^{30}$ Our study extends those results to describe a similar dose-response relationship between previous cigarette exposure and risk of incident HF in older adults, even when adjusted for incidentcoronary events. Although smoking cessation leads to a decrease risk of cardiovascular events and mortality, there is evidence that inflammatory, hemostatic, and atherosclerotic changes can take up to 20 years to revert back to non-smoker levels, underscoring the importance of avoiding smoking. ${ }^{31,32}$ However, this risk in past smokers is still reduced in magnitude in comparison to current smokers, thus underscoring the benefits of smoking cessation.

Current smokers exhibited the greatest HF risk compared to past smokers and non-smokers. Interestingly, no dose-effect association between pack-years of cigarette smoking and risk was observed in this group. This lack of dose-effect is mechanistically conceivable since acute cigarette smoking reduces myocardial flow reserve, ${ }^{33}$ increases reactive oxygen species and platelet hyperactivity, ${ }^{34}$ augments catecholamine release, ${ }^{10}$ and decreases nitric oxide production and endothelium-dependent vasodilation. ${ }^{35}$ These derangements may have persistent deleterious effects on individuals irrespective of duration and amount of exposure, underscoring the fact that there may be no safe level of smoking cigarettes. However, the small sample size of the current smokers, compared to past smokers, must be recognized as an alternative possibility for the lack of a dose-effect association in this group.

This study has several limitations. Smoking data, including pack-years of exposure, rely on self-report by participantsat enrollment in the study. Therefore, the possibility of exposure misclassification cannot be excluded. Echocardiography was not systematically evaluated at index HF hospitalizations and therefore our conclusions about the association between smoking and HF with preserved or reduced left ventricular ejection fraction are potentially subject to bias. In addition, the small number of current smokers, and particularly of those in the lowest tertile of pack years, does not allow for safe conclusions on the association between cigarette exposure and HF risk in this group. 
In conclusion, this study demonstrates an independent association between smoking and the development of incident HF in older adults, irrespective of other risk factors and incident coronary events. There does not appear to be any safe level of smoking in terms of HF risk in older adults. As smoking continues to be a leading cause of preventable morbidity and mortality, continued attempts at the individual and population level to reduce smoking appear warranted.

\section{Acknowledgments}

Funding: This research was supported in part by the Intramural Research Program of the National Institute of Aging (NIA), National Institutes of Health, Bethesda, MD and by NIA Contracts N 01-AG-6-2101; N01AG-6-2103; N01-AG-6-2106; NIA grant R01-AG028050, and NINR grant R01-NR012459. The authors are solely responsible for the design and conduct of this study; all study analyses, the drafting and editing of the paper and its final contents.

\section{References}

1. Ezzati M, Lopez AD. Regional, disease specific patterns of smoking-attributable mortality in 2000. Tob Control. 2004; 13:388-395. [PubMed: 15564623]

2. Barua RS, Ambrose JA, Eales-Reynolds LJ, et al. Dysfunctional endothelial nitric oxide biosynthesis in healthy smokers with impaired endothelium-dependent vasodilatation. Circulation. 2001; 104:1905-1910. [PubMed: 11602492]

3. Cross CE, Halliwell B, Borish ET, et al. Oxygen radicals and human disease. Ann Intern Med. 1987; 107:526-545. [PubMed: 3307585]

4. FitzGerald GA, Oates JA, Nowak J. Cigarette smoking and hemostatic function. Am Heart J. 1988; 115:267-271. [PubMed: 3276116]

5. Mendall MA, Patel P, Asante M, et al. Relation of serum cytokine concentrations to cardiovascular risk factors and coronary heart disease. Heart. 1997; 78:273-277. [PubMed: 9391290]

6. Ambrose JA, Barua RS. The pathophysiology of cigarette smoking and cardiovascular disease: an update. J Am Coll Cardiol. 2004; 43:1731-1737. [PubMed: 15145091]

7. El-Menyar AA. Cytokines and myocardial dysfunction: state of the art. J Card Fail. 2008; 14:61-74. [PubMed: 18226775]

8. Ahmed SS, Moschos CB, Lyons MM, et al. Cardiovascular effects of long-term cigarette smoking and nicotine administration. Am J Cardiol. 1976; 37:33-40. [PubMed: 1244732]

9. Alam M, Samad BA, Wardell J, et al. Acute effects of smoking on diastolic function in healthy participants: studies by conventional doppler echocardiography and doppler tissue imaging. J Am Soc Echocardiogr. 2002; 15:1232-1237. [PubMed: 12411910]

10. Cryer PE, Haymond MW, Santiago JV, et al. Norepinephrine and epinephrine release and adrenergic mediation of smoking-associated hemodynamic and metabolic events. N Engl J Med. 1976; 295:573-577. [PubMed: 950972]

11. Price JF, Mowbray PI, Lee AJ, et al. Relationship between smoking and cardiovascular risk factors in the development of peripheral arterial disease and coronary artery disease: Edinburgh Artery Study. Eur Heart J. 1999; 20:344-353. [PubMed: 10206381]

12. Unverdorben M, von Holt K, Winkelmann BR. Smoking and atherosclerotic cardiovascular disease: part II: role of cigarette smoking in cardiovascular disease development. Biomark Med. 2009; 3:617-653. [PubMed: 20477529]

13. Butler J, Kalogeropoulos A, Georgiopoulou V, et al. Incident heart failure prediction in the elderly: the health ABC heart failure score. Circ Heart Fail. 2008; 1:125-133. [PubMed: 19777072]

14. Bowman TS, Gaziano JM, Buring JE, et al. A prospective study of cigarette smoking and risk of incident hypertension in women. J Am Coll Cardiol. 2007; 50:2085-2092. [PubMed: 18021879]

15. Conen D, Everett BM, Kurth T, et al. Smoking, smoking cessation, [corrected] and risk for symptomatic peripheral artery disease in women: a cohort study. Ann Intern Med. 2011; 154:719726. [PubMed: 21646555] 
16. Doll R, Peto R, Boreham J, et al. Mortality in relation to smoking: 50 years' observations on male British doctors. BMJ. 2004; 328:1519. [PubMed: 15213107]

17. Halperin RO, Gaziano JM, Sesso HD. Smoking and the risk of incident hypertension in middleaged and older men. Am J Hypertens. 2008; 21:148-152. [PubMed: 18174885]

18. Fried LP, Borhani NO, Enright P, et al. The Cardiovascular Health Study: design and rationale. Ann Epidemiol. 1991; 1:263-276. [PubMed: 1669507]

19. Rodondi N, Newman AB, Vittinghoff E, et al. Subclinical hypothyroidism and the risk of heart failure, other cardiovascular events, and death. Arch Intern Med. 2005; 165:2460-2466. [PubMed: 16314541]

20. Wang J, Nagueh SF. Current perspectives on cardiac function in patients with diastolic heart failure. Circulation. 2009; 119:1146-1157. [PubMed: 19255354]

21. Fine JP, Gray RJ. A proportional hazards model for the subdistribution of a competing risk. J Am Stat Assoc. 1999; 94:496-509.

22. Berry SD, Ngo L, Samelson EJ, et al. Competing risk of death: an important consideration in studies of older adults. J Am Geriatr Soc. 2010; 58:783-787. [PubMed: 20345862]

23. Bermudez EA, Rifai N, Buring JE, et al. Relation between markers of systemic vascular inflammation and smoking in women. Am J Cardiol. 2002; 89:1117-1119. [PubMed: 11988205]

24. Bahrami H, Bluemke DA, Kronmal R, et al. Novel metabolic risk factors for incident heart failure and their relationship with obesity: the MESA (Multi-Ethnic Study of Atherosclerosis) study. J Am Coll Cardiol. 2008; 51:1775-1783. [PubMed: 18452784]

25. Kalogeropoulos A, Georgiopoulou V, Psaty BM, et al. Inflammatory markers and incident heart failure risk in older adults: the Health ABC (Health, Aging, and Body Composition) study. J Am Coll Cardiol. 55:2129-2137. [PubMed: 20447537]

26. Orosz Z, Csiszar A, Labinskyy N, et al. Cigarette smoke-induced proinflammatory alterations in the endothelial phenotype: role of NAD(P)H oxidase activation. Am J Physiol Heart Circ Physiol. 2007; 292:H130-139. [PubMed: 17213480]

27. Knight-Lozano CA, Young CG, Burow DL, et al. Cigarette smoke exposure and hypercholesterolemia increase mitochondrial damage in cardiovascular tissues. Circulation. 2002; 105:849-854. [PubMed: 11854126]

28. Hellerstein MK, Benowitz NL, Neese RA, et al. Effects of cigarette smoking and its cessation on lipid metabolism and energy expenditure in heavy smokers. J Clin Invest. 1994; 93:265-272. [PubMed: 8282797]

29. Baldassarre D, Castelnuovo S, Frigerio B, et al. Effects of timing and extent of smoking, type of cigarettes, and concomitant risk factors on the association between smoking and subclinical atherosclerosis. Stroke. 2009; 40:1991-1998. [PubMed: 19359639]

30. Rosen BD, Saad MF, Shea S, et al. Hypertension and smoking are associated with reduced regional left ventricular function in asymptomatic: individuals the Multi-Ethnic Study of Atherosclerosis. J Am Coll Cardiol. 2006; 47:1150-1158. [PubMed: 16545644]

31. Critchley JA, Capewell S. Mortality risk reduction associated with smoking cessation in patients with coronary heart disease: a systematic review. JAMA. 2003; 290:86-97. [PubMed: 12837716]

32. Woodward M, Lam TH, Barzi F, et al. Smoking, quitting, and the risk of cardiovascular disease among women and men in the Asia-Pacific region. Int J Epidemiol. 2005; 34:1036-1045. [PubMed: 15914503]

33. Czernin J, Sun K, Brunken R, et al. Effect of acute and long-term smoking on myocardial blood flow and flow reserve. Circulation. 1995; 91:2891-2897. [PubMed: 7796497]

34. Blache D. Involvement of hydrogen and lipid peroxides in acute tobacco smoking-induced platelet hyperactivity. Am J Physiol. 1995; 268:H679-685. [PubMed: 7864194]

35. Barua RS, Ambrose JA, Eales-Reynolds LJ, et al. Heavy and light cigarette smokers have similar dysfunction of endothelial vasoregulatory activity: an in vivo and in vitro correlation. J Am Coll Cardiol. 2002; 39:1758-1763. [PubMed: 12039488] 


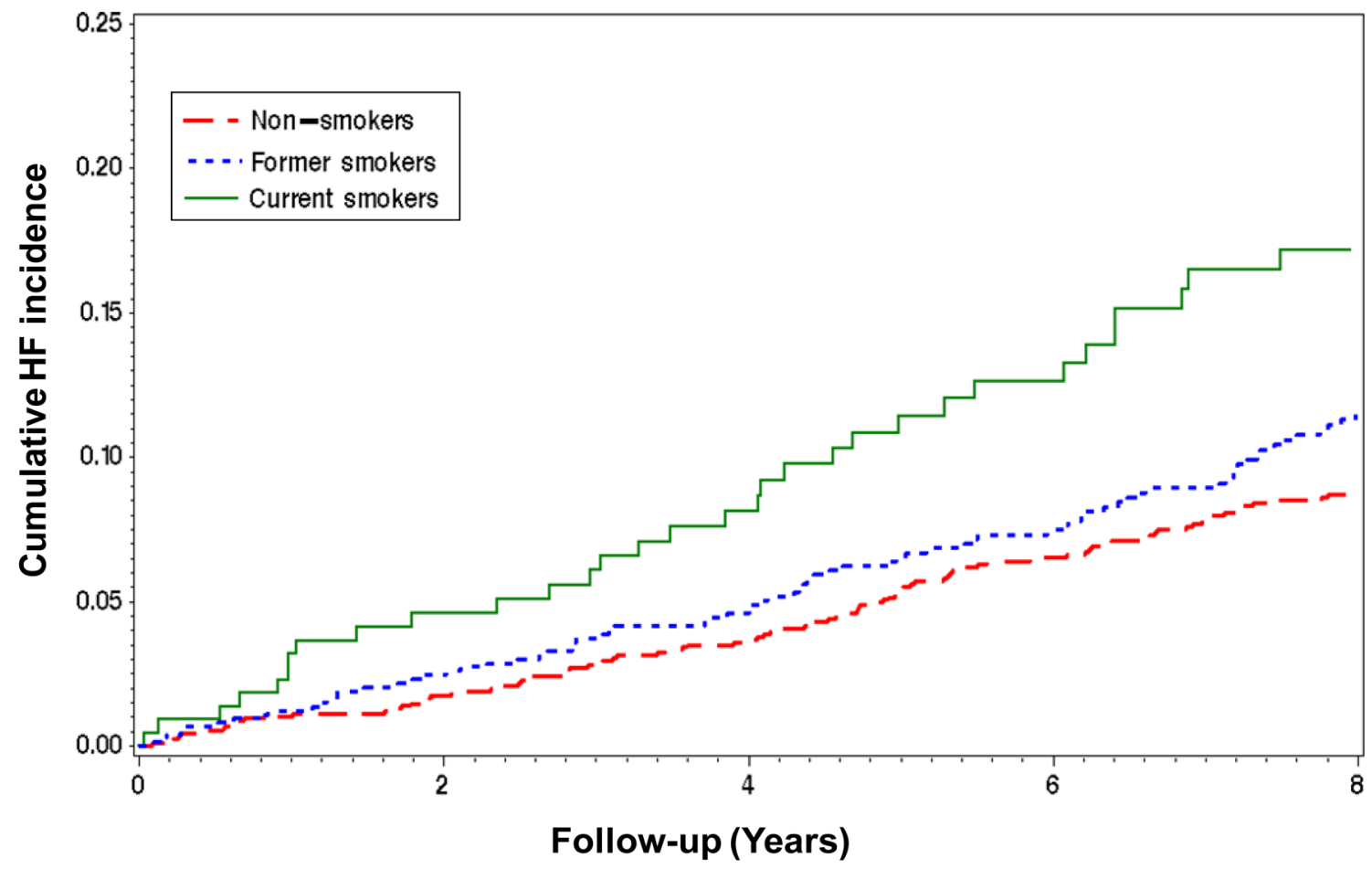

Figure 1. Kaplan-Meier Curves by Smoking Status

Cumulative heart failure incidence by smoking status at inception in the Health ABC Study 


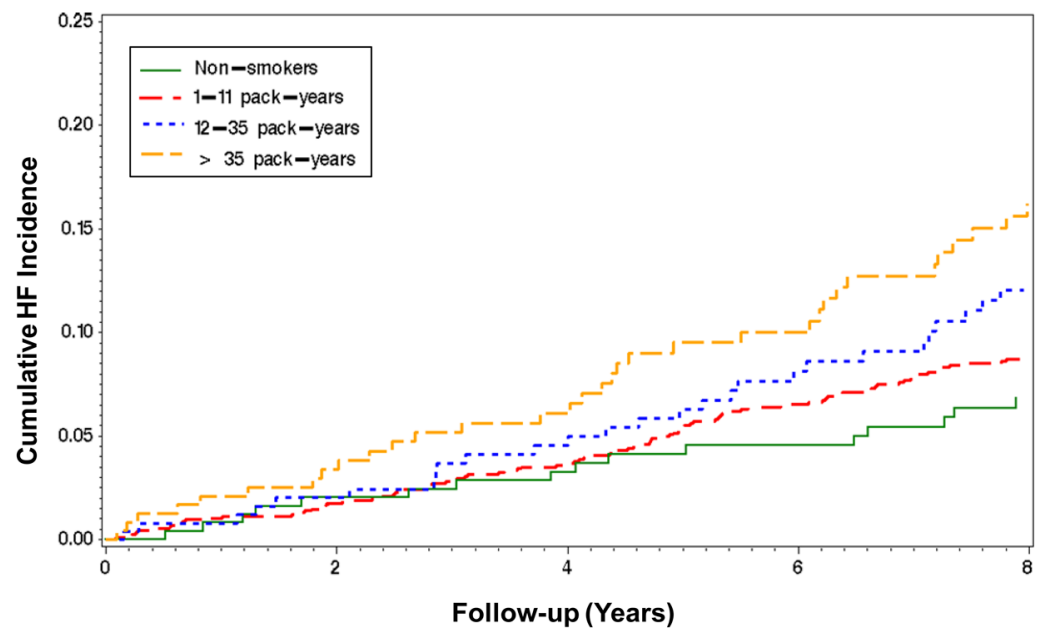

Figure 2. Kaplan-Meier Curve of Past Smokers

Cumulative heart failure incidence over time according to tertiles of pack-years of smoking exposure among past smokers in the Health ABC Study. Incidence among non-smokers (green line) is also shown for comparison. 


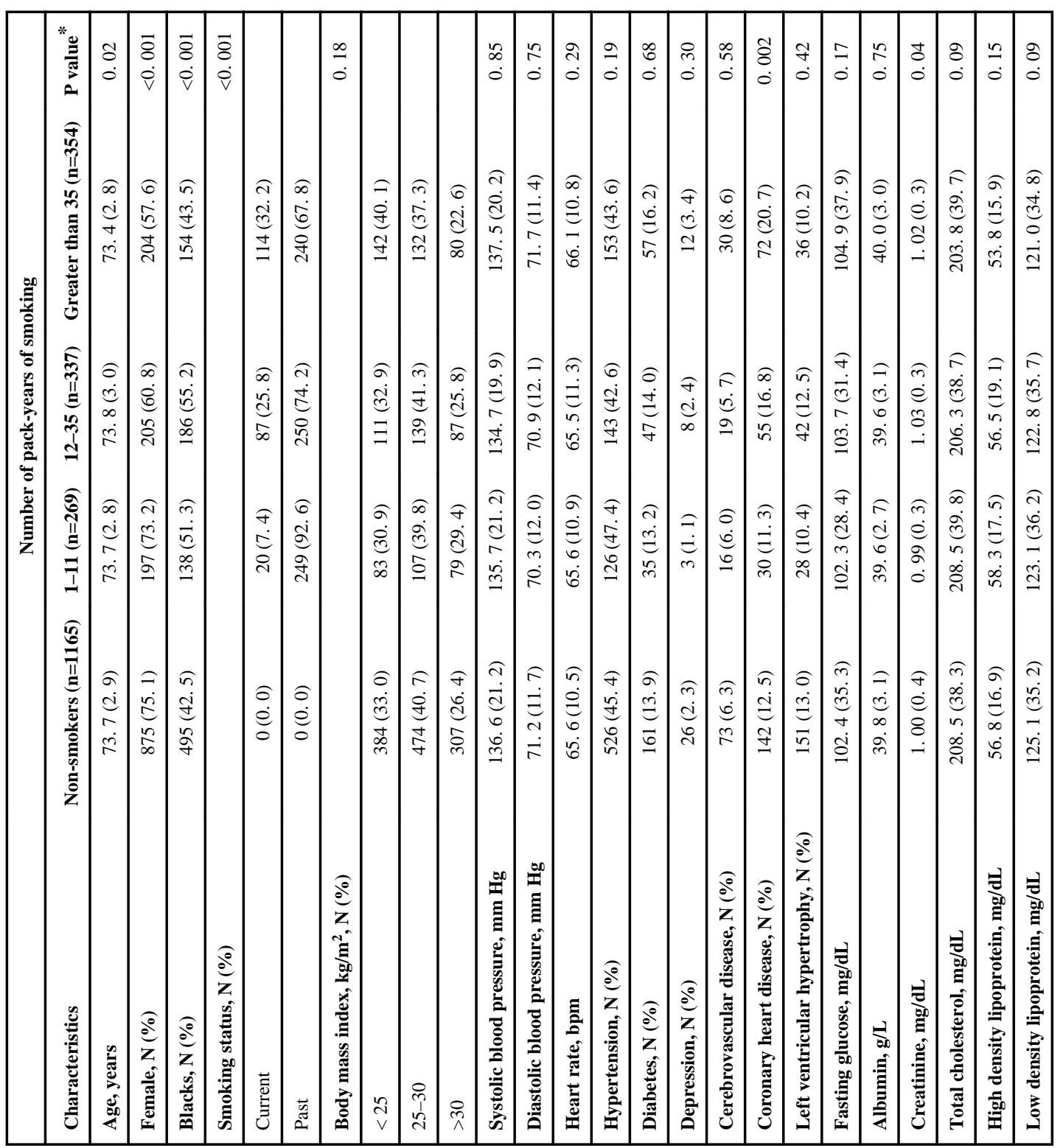




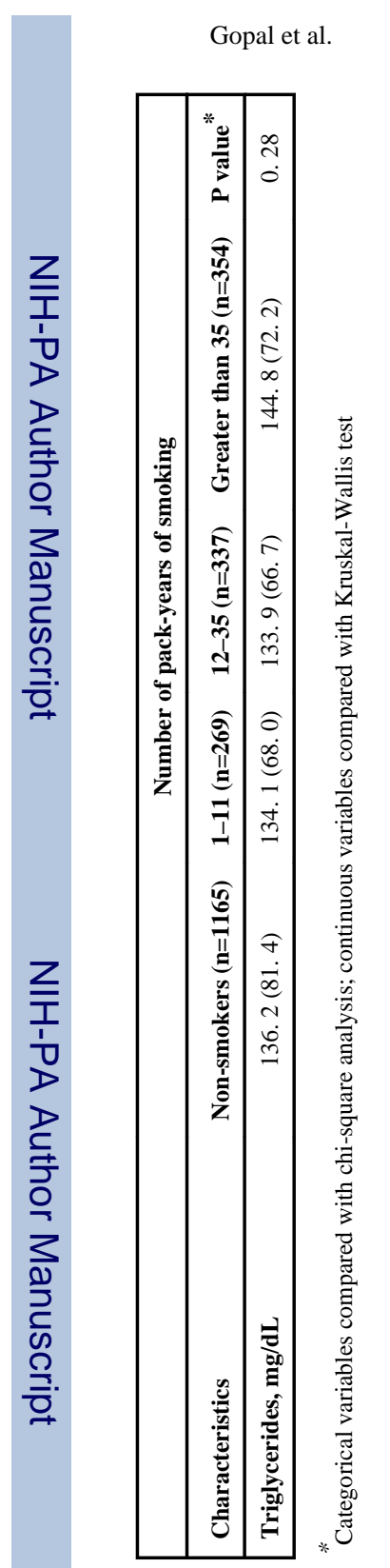

Page 12

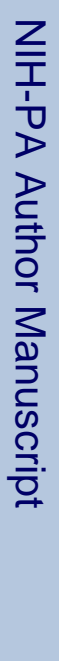

Am Heart J. Author manuscript; available in PMC 2013 August 01. 


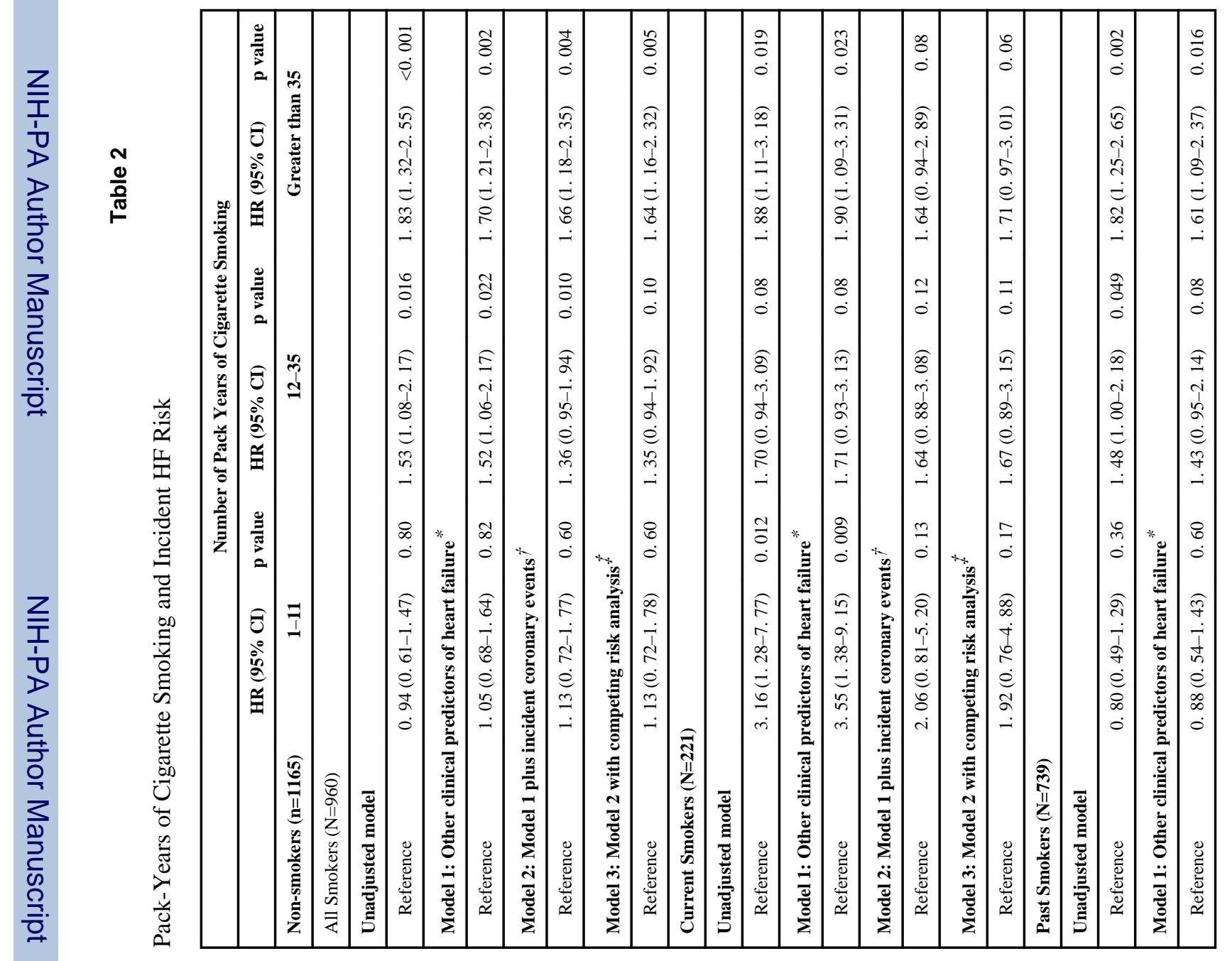




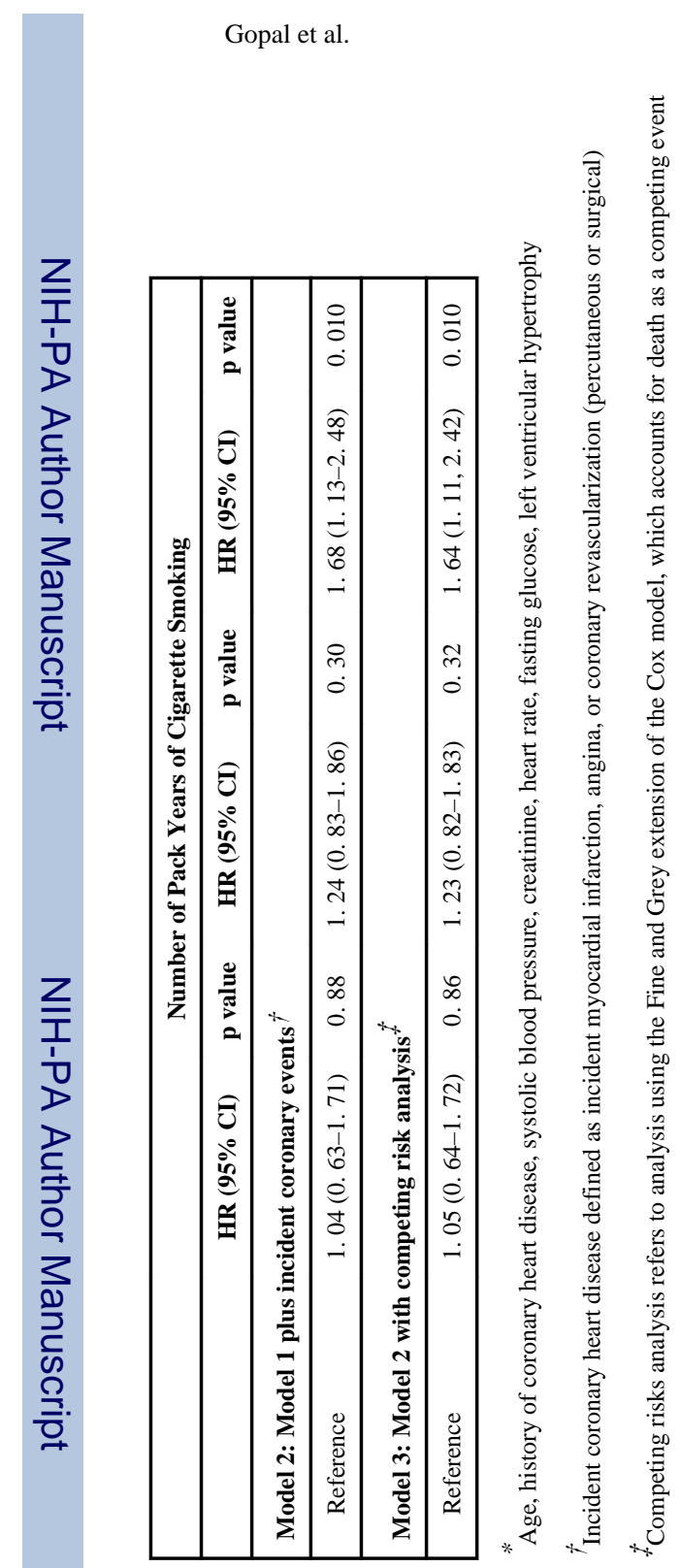

Page 14

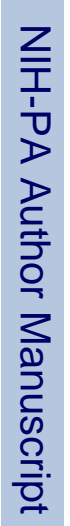

Am Heart J. Author manuscript; available in PMC 2013 August 01. 